\title{
Design and Implement of VHF Simulator Based on Cross Platform
}

\author{
Li Shaowei ${ }^{1+}$ \\ ${ }^{1}$ School of Mathematics and Computer Science, Jianghan University, Wuhan, Hubei, China
}

\begin{abstract}
The data transmission efficiency of existing marine VHF voice communication simulators is low and they are only single-function simulators. In order to improve the shortcomings mentioned above, a crossplatform dual-purpose (ship and land use) VHF simulator was designed in this pater. The voice signal is captured and played back by the computer's sound card and then, the voice data is compressed with wavelet algorithm. Data sending and receiving are completed via computer networks and ports instead of radio communication channels. The software of this simulator runs on different operating system, because it was developed based on cross-platform tool Qt. Function multiplexing of hardware and software were achieved to decrease development cost.
\end{abstract}

Keywords: VHF, simulator, voice data, wavelet algorithm, cross platform

\section{Introduction}

Marine VHF is one of the most important way in maritime voice communication, it is also an important teaching aid in maritime simulator education. However, each VHF terminal exchanges information with radio, which may cause interference and bring out terrible disasters. To overtake the disadvantage of real VHF in education, the VHF simulator that does not emit radio was invented. In current time, it has become the standard component in maritime education [1-3].

$\mathrm{Yu}$ Lili in Shanghai Maritime University designed marine VHF based on internet with voice processing chip [4] The chip integrates the functions of voice signal gathering and processing, after data acquiring, it transmits information to PC via RS232 interface, and then broadcasts via internet.

On one hand, the cost increased due to additional hardware applied; on the other hand, RS232 is a low baud rate interface and becomes the bottleneck of data channel. Yu decided the baud rate to be 19200bps according to his paper, so, in the situation of mono and 8bit per channel, its maximum band width can be figured out by formula (1)

$$
W=\frac{19200}{8}=2400(H z)
$$

The frequency of voice is always considered as $3.4 \mathrm{KHz}$, based on nyquist sampling law, the system obviously cannot satisfy actual demand due to equation (1). Ding Feng from Shanghai Ocean University developed the VHF simulator based on Flash [5], but his research focused on theory and the realization methods was not mentioned. From his conclusion, we could not know about the effect of his system. Moreover, VHF simulator that was developed with pure Flash software lacks of realism and influences teaching effectiveness.

To enhance the transmission efficiency of VHF simulator and reproduce real scene, a kind of dualpurpose marine VHF simulator based on cross platform was proposed. Its prototype is the Vessel Transport Service (VTS) system whose model is ATLAS 9760 and it has been applied in project VTS Simulator System in Shanghai Maritime Security Administration. At the top level of hardware designing, multiplexing

+ Corresponding author. Tel.: + 86-18171385977; fax: +86-2785824007.

E-mail address: swli@jhun.edu.cn. 
function for ship and land station use was achieved. Only few hardware for human interface was added since the system takes advantage of existing computer and network resources; In terms of software, source code can be complied on different platforms because it was programed with Qt who is a cross-platform development tool. The architecture of VHF simulator is shown as figure 1 below.

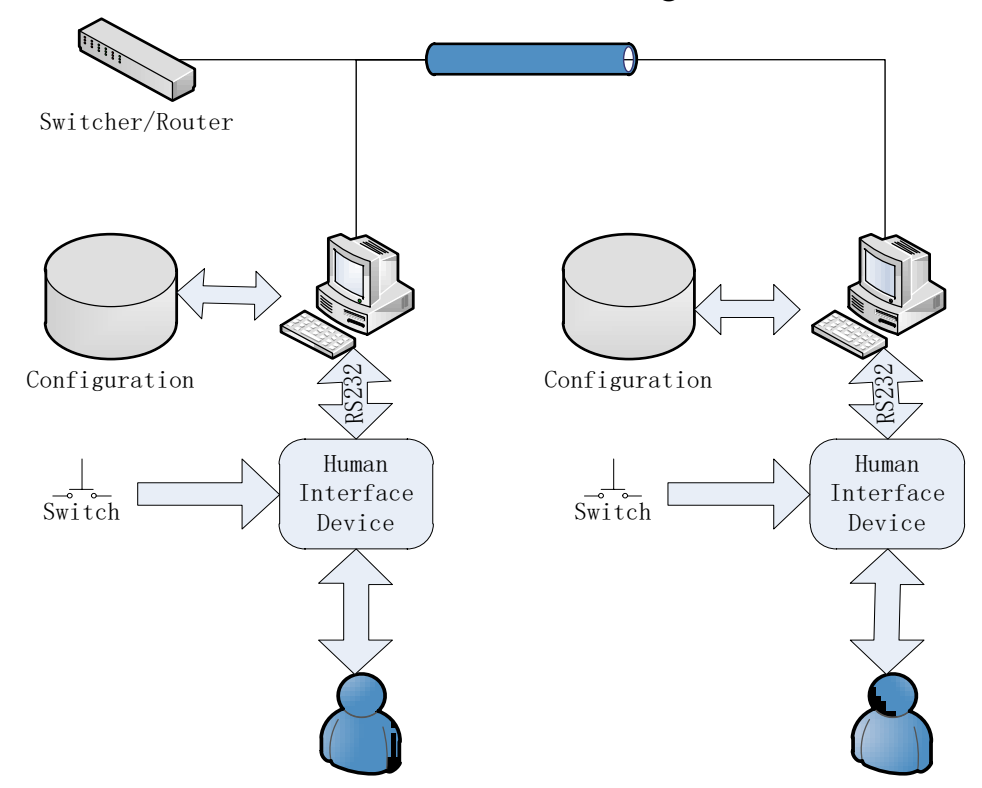

Fig. 1: Architecture of VHF simulator

\section{VHF Simulator System}

VHF simulator is composed of human interface device, PC, configuration file and networks. The core unit of HID is micro control unit (MCU) [9], it has the advantage of low cost and easy to use. It monitors the action of operators all the time and sends action codes to PC via RS232; PC, whose main function is to acquire information and transmit-receive voice data according to configuration files [6]. Figure 2 shows the working flow diagram of simulator system.

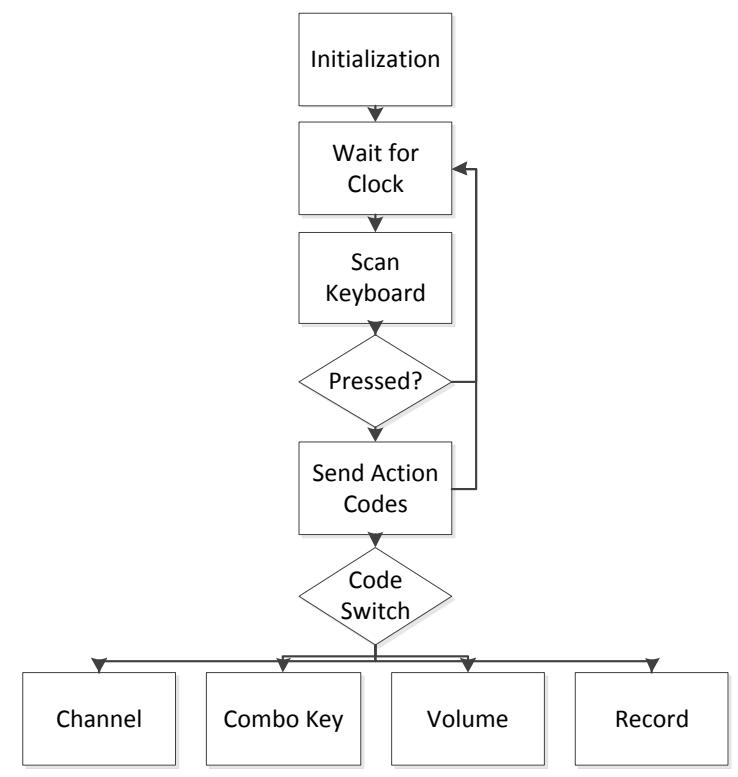

Fig. 2: Working flow of simulator

From figure 2 we can see that, MCU is driven by clock pulse and scans keyboard periodically, once the key was pressed, it encodes action information and sends to PC; then, PC gives response and invokes related handlers.

\section{Key Technology on VHF System}




\subsection{Hardware structure and multiplexing}

$80 \mathrm{C} 51$ serial MCU was chosen as core processor, and the hardware consists of switch, matrix keyboard, volume control module, LCD and serial communication module. Figure 3 shows the structure of hardware.

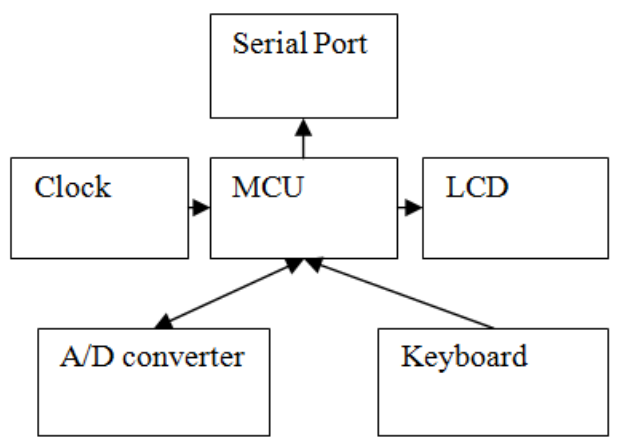

Fig. 3: Structure of hardware

The figure above shows that, the simulator system is composed of 5 components and all these parts can be commonly used in both marine and land station VHF simulator with little differences such as appearance and number of keyboard. Therefore, we could design a kind of dual-purpose VHF simulator system in relative easy way. The components' functions of VHF simulator can be described as below:

(1) MCU. It is the core control unit of the whole system.

(2) Serial communication. It is the data channel for human interface and its baud rate is set $9600 \mathrm{kbps}$.

(3) Display module. It shows the status of the system to user in graphic way.

(4) Keyboard. It is used to gather user's operation and select corresponding function. The land station VHF simulator keyboard has 32 keys and is implemented in matrix keyboard method with 12 pins; meanwhile, the marine VHF simulator keyboard only has 6 keys. To keep the consistency of PCB in different system, 26 more keys are shield in marine VHF system.

(5) A/D converter. A/D module monitors the value of potentiometer as it is chosen to adjust system volume

\subsection{Voice Acquiring and Playing}

As to reduce the cost of the system, we took advantage of sound adapter in PC to achieve voice acquiring and playing ${ }^{[7]}$. Figure 4 illustrates us the working flow of voice data processing.

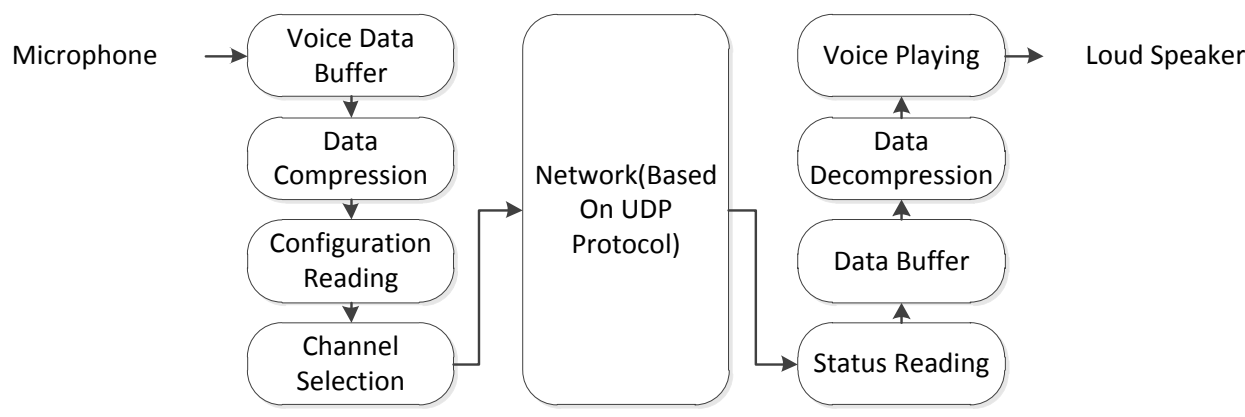

Fig. 4: Acquiring and playing process of voice data

Voice acquiring and playing interface has been built in cross-platform development tool Qt. The names of the API are QAudioInput and QAudioOutput [8] respectively. We need to set proper parameters prior to applying the interface in program. Table 1 shows us the parameters in this system.

Table 1: $\quad$ Sample parameter of voice data

\begin{tabular}{|l|l|}
\hline Sample Rate & $44.1 \mathrm{KHz}$ \\
\hline Sound Channel & Stereo \\
\hline Resolution & $8 \mathrm{bit}$ \\
\hline Buffer Size & $8 \mathrm{~KB}$ \\
\hline Number of Buffers & 4 \\
\hline
\end{tabular}




\section{Format}

PCM

Multi-buffer technology is applied in the system, once the current buffer was full, backup buffer could be switched instantly. Figure 5 demonstrates the buffer switching with 4 buffers.

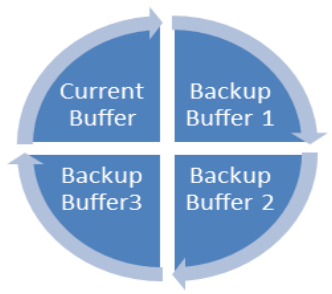

Fig. 5: Buffer for voice data

\subsection{Serial communication module on cross-platform}

Since the serial communication interface has not been achieved in $\mathrm{Qt}$, we have developed different communication programs on different platforms and compiled them as dynamic link library (DLL), which is supported by all modern operating systems. So, we can invoke related functions with minor modification.

In different DLLs, all the function interfaces are led out with the same names. Macro definition \#define in $\mathrm{C}$ language was used to distinguish different operating systems. The table below tells the interface of functions.

Table 2: General functions for serial port

\begin{tabular}{|l|l|l|l|l|}
\hline Open & Setting & Read & Write & Close \\
\hline OpenPort () & SetPort () & ReadPort () & WritePort () & ClosePort () \\
\hline
\end{tabular}

Function OpenPort accepts one parameter and was used to identify serial port number; Function SetPort was used to set baud rate, it accepts one integer variable; Function ReadPort/WritePort was designed to read and write serial port in blocking way, so, these two functions need to run in an independent thread.

\subsection{Voice data compression}

In local area networks, it is not necessary to compress voice data due to wide band and low network latency. However, once the system is applied in wide area networks, voice data needs to be compressed prior to transmitting.

In this simulator system, wavelet algorithm was brought out to compress voice data, its definition is shown as below

$$
W(f)_{a, b}=\int_{R} f(x) \overline{\Psi a, b}(x) d x
$$

where

$$
\Psi_{a, b}(x)=\frac{1}{\sqrt{a}} \Psi\left(\frac{x-b}{a}\right)
$$

is called wavelet basis function. Here we proposed Harr orthogonal wavelet basis to decompose sample voice data with three level wavelet packet, and then reconstructed.
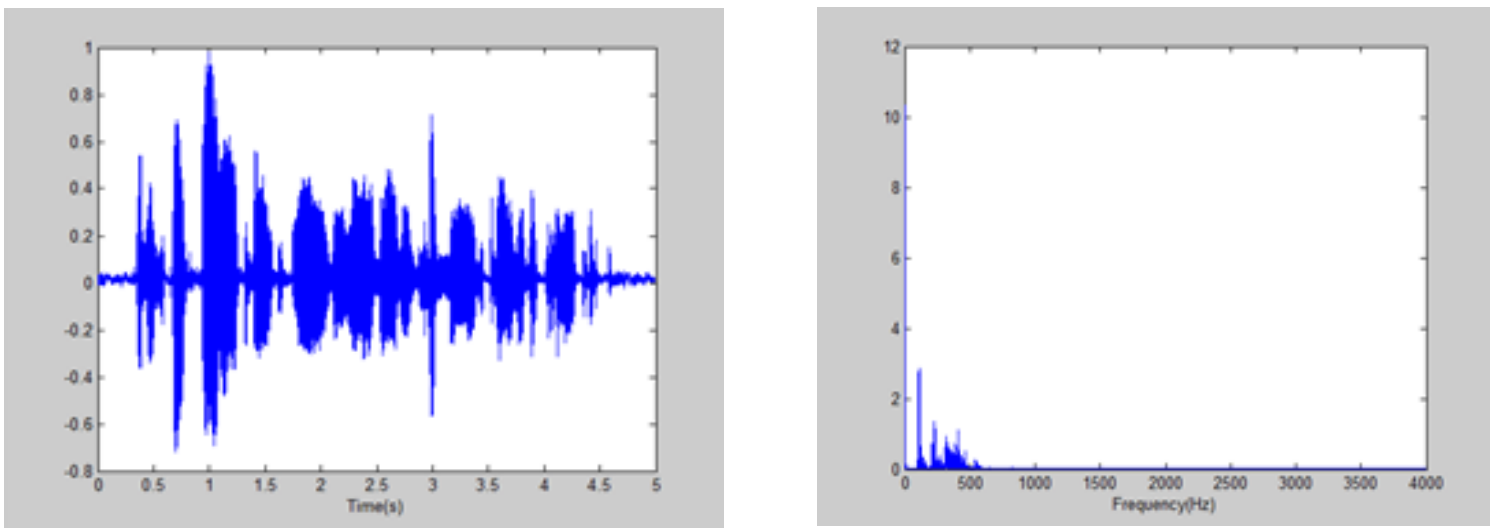

Fig. 6: Raw voice data in time/frequency domain 

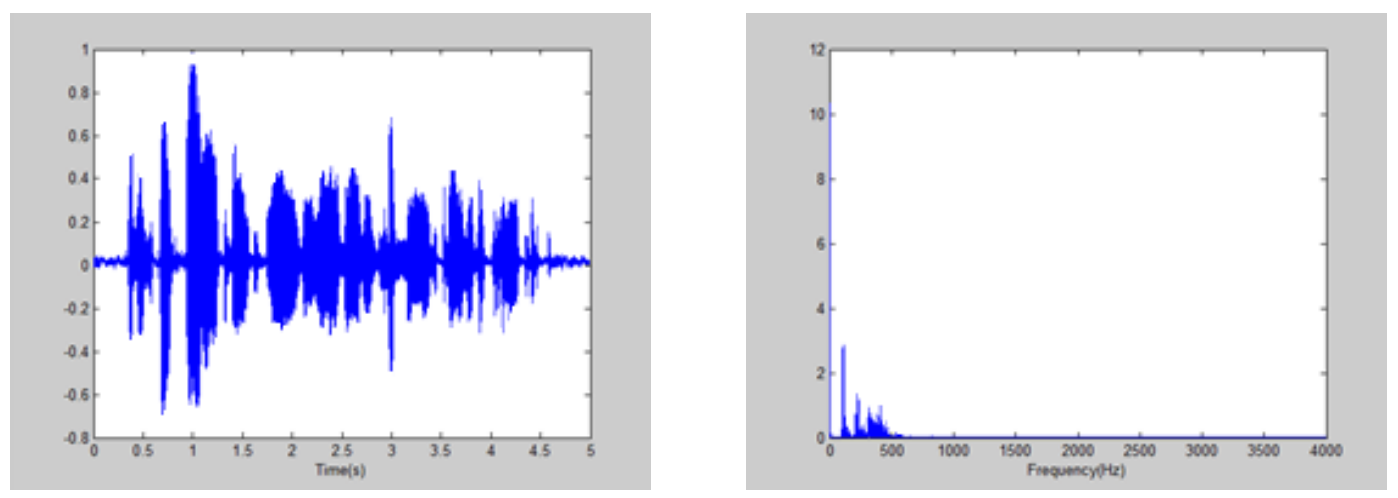

Fig 7: Reconstructed voice data in time/frequency domain

Figure 6 above shows us the original voice sample in time/frequency domain; Figure 7 ilustrates the voice data after reconstruction. From these figures, we can see that there is little distortion in high frequency field and human can barely distinguish.

The compression rate is defined as below:

$$
N=\frac{B}{A} \times 100 \%
$$

Symbol B represents compressed data and A means original data. For the sample voice signal, its compression rate can be calculated through the formula and the value is about 0.515 . It is not necessary to increase data volume in higher frequency part due to more data belongs to low frequency and it has merely influence on compression rate.

\section{Conclusion}

The whole simulator system consists of land station and ship borne VHF. Figure 8 demonstrates the running result of VHF simulator, where the shore-based VHF is used by maritime administration unit and ship borne VHF is always treated as training equipment. The system can meet the most requirements of navigation training combining with two equipment. Moreover, this simulator system can run on Windows, WinCE and Linux with little code changing, because it is developed on cross-platform tool Qt [11-13].

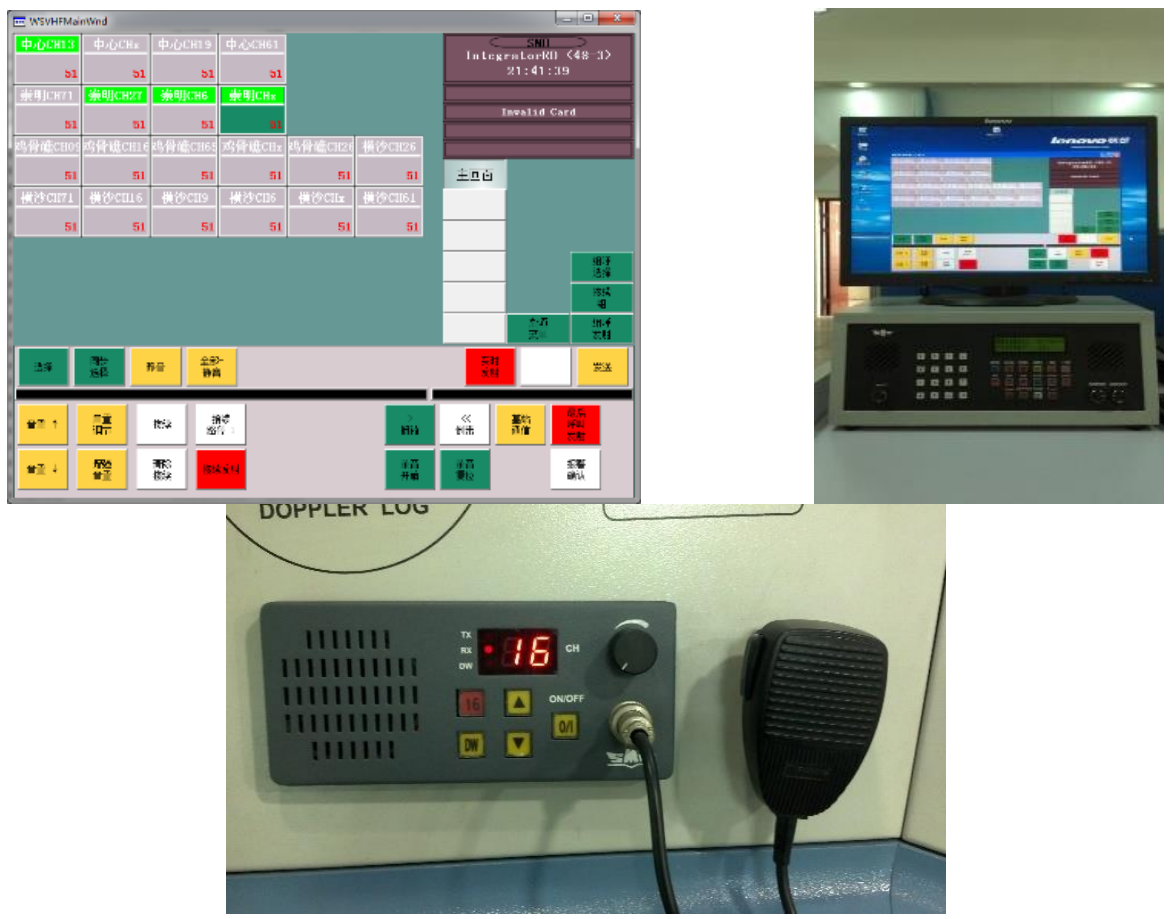

Fig. 8: Realization of Simulator 


\section{References}

[1] Shi Chaojian, Chen Jinbiao, Hu Qinyou. Global networking for development and application of shiphandling simulators [J]. Journal of Shanghai Maritime University, 2007, 28(1):1-6.

[2] Shi Chaojian, Hu Shenping, Chen Jinbiao. Study on performance standard of shiphandling simulator [J]. Journal of Shanghai Maritime University,2005,26(2):4-8.

[3] Liu Jianping, Huang Yong, Zhou Xiliu. Cloud Computing Science and Technology Information Service System Platform Design[J]. Bulletin of Science and Technology, 2012,10(28):19-21

[4] Yu Lili, Shi Chaojian, Huang Zhenmin et al. Ship's VHF simulator system integrated by multiple simulators [J]. Journal of Shanghai Maritime University, 2009,30(1):20-23

[5] Ding Feng, Wei Wei. The Realization of Voice Communication of the VHF Simulator Based on Flash Media Server[J]. Journal of Qingdao Ocean Shipping Mariners College, 2009,30(4):9-11

[6] Lv Junya. Design and Implementation of Temperature Controlling System Based on Single Chip[J]. Computer Simulation, 2012,29(7):230-233.

[7] Li Shaowei, Zhuang Xinqing. Modules of voice and data transmission based on Inmarsat-F[J]. Navigation of China, 2008,31(2):114-134.

[8] Jasmin Blanchette, Mark Summerfield. C++ GUI Programming with Qt 4 (2nd Edition)[M].Canada: Prentice Hall PTR,2008

[9] Zhang Xijun, Wang Jizeng, Lin Ying. ech and Data Compression on Wavelet Packet Transformation[J]. Science Technology and Engineering, 2009,9(2):272-276.

[10] Cui Jing, Wang Xuemei. Research of Application and Simulation for Image Compression Algorithm[J].Computer Simulation,2012,29(8):265-268.

[11] Wang Shengzheng, Shi Chaojian, Shi Yonghui. New key techniques for navigation simulator[J]. Journal of Shanghai Maritime University, 2007,28(1):143-149

[12] Duane Hanselman, Bruce Littlefield. Mastering Matlab 7[M].Beijing: Tsinghua University Press, 2006

[13] Liao Shuhe. Analysis of System Composition of Full Mission Shio Handling Simulator[J]. Navigation of China,2003,55(2):8-17

[14] Zeng Qingjun, Zhou Yaoting. Study on Key Content of Integrated Bridge System[J]. Marine Technology,2001(4):24-26 